\title{
INFLUENCIA DA GESTAÇÃO E DO PUERPÉRIO SOBRE O QUADRO HEMATICO DE BOVINOS DA RAÇA HOLANDESA BRANCA E PRETA
}

\author{
José Luiz D'ANGELINO * \\ Leonardo Miranda de ARAUJO *** \\ Eduardo II. BIRGEL \\ Wanderley Pereira de ARAUJO * \\ Carlos Eduardo REICHMANN **
}

\begin{abstract}
RFMV-A/1
D'ANGELINO, J.L.; ARAUJO, L.M.; BIRGEL, E.H.; ARAUJO, W.P.; REICHMANN, C.E. Influência da gestaçðo e do puerpério sobre o quadro hemático de bovinos da raça Holandesa branca $e$ preta. Rev. Fac. Med. vet. Zootec. Univ. S. Paulo, 14/1):11.21, 1977.
\end{abstract}

RESUMO: As variaçōes do quadro hemático de bovinos em gestação foram estudadas em 75 novilhas da raça holandesa branca e preta. No final da gestação observou-se um aumento estatisticamente significante do volume médio de hemácias (VCM), responsével direto pelo maior valor do hematócrito, no mesmo periodo. De maneira contrória, por permanecerem inalteradas as taxas de hemoglobina, observou-se uma diminuição estatisticamente significante da concentraçäo de hemoglobina corpuscular média (CHCM) no final da gestacäo. A contagem global de leucócitos e os diferentes tipos destas células näo sofreram influência estatisticamente significantes da gestaçâo e do puerpério. Os resultados obtidos para o grupo das novilhas vazias, de animais na fase inicial da gestação, entre 4 e 6 meses de gestaçāo, de novilhas na fase final da gestaçäo e vacas durante o puerpério, foram respectivamente.

Hemácias $\times 10^{6} \mathrm{~mm}^{3}-6,01 ; 5,89 ; 5,95 ; 5,95$ e 6,3 ?

hemoglobina $\mathrm{g} \%$ - 9,63;10,22;10,24; 9,85 e 9,58

hematocrito $\%$ - 27,52; 28,$79 ; 29,05 ; 31,62$ e 28,93

vol. cop. médio $\mu^{3}-46,43 ; 49,41 ; 49,17 ; 53,57$ e 44,63

hemoglob. corp. média $\gamma \gamma-16,27 ; 17,47 ; 17,28 ; 16,82$ e 15,18

conc. Hemoglob. corp. média \% - 34,63; 35,15;35,20;31,13 e 33,03

Leucócitos $\times 10^{3} \mathrm{~mm}^{3}-16,17 ; 18,45 ; 16,14 ; 18,03$ e 15,15

Neutrófilos \% - 21,7; 24,3; 19,1; 18,0 e 24,8

$\mathrm{mm}^{3}-3528,7 ; 4505,7 ; 3026,1 ; 3198,5$ e 3616,6

Eosinofilos $\begin{gathered}\% \\ \mathrm{~mm}^{3}-625.7 ; 734,5 ; 803,9 ; 925,3 \text { e 605,3 }\end{gathered}$

Basofilos $\begin{array}{r}\% \\ \mathrm{~mm}^{3}-0 ; 0,1 ; 0,2 ; 0,1 \text { e } 0,1 \\ \text { - } 0 ; 15,3 ; 33,6 ; 22,1 \text { e } 19.7\end{array}$

Linfócitos - 72,4; 67,9; 74,5; 74,9 e 69,1

Linfocitos $\mathrm{mm}^{3}-11673,6 ; 12894,3 ; 12067,8 ; 13524,6$ e 10650,2

Monócitos \% - 2,1; 1,3; 1,3; 2,0 e 1,8

$\mathrm{mm}^{3}-351,0 ; 281,9 ; 209,0 ; 355,3$ e 261,5

UNITERMOS: Hematologia *; Prenhez, bovinos *; Puerpério; Quadro hemático.

* Auxiliar de Ensino.

* * Professor Assistente Doutor.

* * Professor Livre-Docente.

Departamento de Patologia e Clínica Médicas da Faculdade de Medicina Veterinária e Zootecnia da USP. 


\section{INTRODUÇĀO}

A hematologia veterinaria tem ap esentado nos últimos anos evidentes e marcantes progressos no sentido de se conhecer os elementos constituintes do sangue dos animais, como também, procurar elucidar os fatores que causam sua variabilidade, entre os quais, salientam-se as condiçoes ambientais e regionais, o tipo de criação e alimentação, além de variações ligadas ao sexo, à idade e eventualmente à raça. Somente após o estabelecimento da amplitude de variação dos resultados devido às referidas causas é que se estabelece um parâmetro regional normal podendo-se assim comparar esses dados, com os resultados em animais acometidos de enfermidades.

Apesar de encontrarmos na literatura muitos trabalhos referentes a hematologia clínica veterinaria, o campo é ainda fértil para novas pesquisas, pois reportando-nos à bibliografia nacional, nåo se encontram pesquisas sistemáticas sobre as variaçoes do quadro sanguíneo de fêmeas bovinas, nos diferentes períodos da gestação, por este motivo é que resolvemos fazer um estudo do quadro hematológico. Assim sendo, a presente pesquisa tem como escopo, de um lado uma contribuição à hematologia veterinária e por outro uma orientação ao clínıco por estabelecer padrões hematológicos regionais.

Apesar de muitos trabalhos encontrados, verificamos que os autores não realizaram um estudo completo, salientando apenas um ou outro elemento do eritrograma. MORRIS 10 (1944), ao estudar o comportamento do numero de hemácias e dos teores de hemoglobina em uma vaca da raça Shorthorn, durante a gestaçāo e parto, constatou ligeira diminuição dos eritrócitos na segunda metade da gestação, sendo esta diminuição mais evidente 36 horas após o parto, havendo normalização destes níveis 15 dias após o evento, salientando ainda que os teores de hemoglobina mantiveram-se sempre constantes durante toda a fase gestacional.

Estudando os efeitos da gestação e da lactação sobre o quadro hemático de vacas primíparas, REYNOLDS ${ }^{13}$ (1953) constatou um aumento do volume sanguíneo, embora o hematócrito permanecesse normal.
Por seu turno, PATTERSON et alii ${ }^{12}$ (1960) preconizaram que os níveis de hemoglobina sofrem efeito da gestação e da lactação; ELECKO ${ }^{5}$ (1967), também encontrou diminuição do número de hemácias e dos teores de hemoglobina em vacas no final da gestação, durante o parto e no puerpério.

Contrariando estes resultados, CONNER et alli $^{4}$ (1967), trabalhando com vacas adultas, evidenciaram năo haver altera ções do hematócrito, salientando porém aumento considerável do numero de hemácias com o progredir da gestaçăo.

Mais recentemente, LANE \& CAMPELL $^{9}$ (1969), em 236 vacas em gestação, notaram ser altamente significante os efeitos da gestaçao sobre o hematócrito, pois verificaram o aumento desse índice, a partir do 6? mês de gestaçăo; resultados estes também constatados por VAN SOEST \& BLOSSER $^{20}$ (1954), salientando no entanto, haver diminuição paulatina dos valores do hematócrito no puerpério.

Estudos completos do quadro leucocitário foram realizados por diversos autores, destacando-se os trabalhos de Moberg (1955), citado por SCHALM ${ }^{17}$ (1964), observou que o numero te leucócitos aumenta até o quarto mês de gestação para, a partir daí, dımınuir. Salienta ainda este autor um comportamento irregular dos neutrófilos que sofre no momento do parto, e diminuição dos linfócitos a partir do $3^{\circ}$ ' més, bem como, oscilações dos eosinófilos durante to do o periodo da gestação.

Entretanto, contrariando o primeiro, STRAUB et alii ${ }^{19}$ (1959), encontraram, durante o parto um aumento considerável do número de leucócitos, neutrófilos, linfócitos e monócitos e uma diminuição de eosinófilos, sendo este resultado semelhante aos obtidos. nas condiçōes já salientadas por OTSUKA \& NAKAJIMA ${ }^{11}$ (1961) e BOSTEDT \& BERCHTOLD ${ }^{3}$ (1968). Em relação aos valores de eosinófilos, KHAJURIA \& RAZDAN $^{8}$ (1966), constataram que o nímero desses elementos do quadro leucocitá rio não sofre absolutamente influencia $\mathrm{da}$ gestação

CONNER et alii ${ }^{4}$ (1967), trabalhando com vacas prenhes, observaram diminuiçāo do número de leucócitos e neutrófilos, não 
constatando variações em relação aos linfócıtos e cosinófilos,

ELECKO $^{5}$ (1967), estudando o comportamento do quadro leucocitário de vacas, antes, durante o parto e no puerpério verificou um aumento gradual do número de leu. cócitos e neutrófilos somente $\mathbf{1 5}$ dias após o parto; já os linfócitos e eusinófilos comportaram-se contrariamente.

\section{MATERIAL E MÉTODOS}

Foram estudadas 75 amostras sanguíneas de bovinos (Bos taurus) fêmeas, da raça holandesa branca e preta, puros de origem, com idades compreendidas entre 15 e 33 meses, não prenhes, em diferentes fases de gestação e no puerpério.

Com o fito de melhor avaliarmos os resultados. procuramos homogeneizar a amostragem, de tal sorte que no presente experimento os animais eram submetidos a iguais condições de manejo e de alimentaçāo Para evitarmos uma possível influência da lactação sobre o quadro hemático utilizamos apenas animais de primeira cria.

Visando uma melhor distribuição dos efeitos da gestação sobre a crase sanguínea, os animais utilizados na presente pesquisa fo. ram divididos em 5 grupos, cada um formado por 15 animais, que ficaram assim constituidos: grupo I - composto de fêmeas não prenhes, cuja idade variava de 15 a 22 meses; grupo II - constituído por fêmeas na fase inicial da prenhez isto é, com até 3 meses de gestação, subdivididas em 3 lotes de 5 animais, com aproximadamente 1, 2 e 3 meses de gestação; grupo III - composto por fêmeas prenhes, cujo período de gestação variava entre 4 e 6 meses, subdivididos em lotes de 5 animais com aproximadamente 4, 5 e 6 meses de gestacâo; grupo IV - formado por fêmeas no perıodo final da prenhez, ou seja entre 7 e 9 meses, subdivididos em lotes de 5 animais, com aproximadamente, $7,8 \mathrm{e}$ 9 meses de gestação; grupo V - constituído por fêmeas recém paridas, subdivididas em lotes de 5 animais com aproximadamente $\mathbf{1}$, 2 e 3 semanas de puerpério.

Antes da coleta, os animais eram submetidos a exame clínico, sendo incluídos no experimento somente aqueles considerados clinicamente sãos.

Por punção da veia jugular, usando-se agulhas apropriadas, recolhíamos $5 \mathrm{ml}$ de sangue, em frascos contendo $0,05 \mathrm{ml}$ de uma solução aquosa a $10 \%$ de EDTA (sal dissódico do ácido etileno-diamino tetracético) correspondendo a $5 \mathrm{mg}$ do sal para $5 \mathrm{ml}$ de sangue conforme recomenda ROSEN. FELD $^{15}$ (1955).

O transporte de todo o material a ser analisado foi realizado logo após a coleta, sendo os mesmos acondicionados em recipientes de isopor, mantidos refrigerados, realizando.se as provas nas 24 horas que se seguiram à coleta.

0 sangue após agitação mecânica durante 2 minutos em aparelho de "Kahn", era coletado em pipetas e a seguir diluído a 1/200 em líquido de Gower, procedendo-se então a contagem das hemácias, em hemacitômetro de Neubauer modificado, segundo a técnica usualmente empregada.

A do agem de hemoglobina foi feita sob a forma de cianometahemoglobina, empregando-se o l'quido de Van Kampen e Zijlstra, segundo técnica indicada por KAMPEN \& ZIJLSTRA ${ }^{7}$ (1963). Os resultados foram obtidos empregando-se uma curva padrão estabelecida a partir de uma amostra sanguínea padronizada, segundo as recomendaçōes de BOROVICZENY² (1963).

0 volume globular foi determinado pelo método de microhematócrito, usando-se os tubos capilares vedados com plastilina e centrifugados a 13.000 r.p.m. durante $5 \mathrm{mi}$ nutos, sendo assim submetidas as amostras a uma força real de centrifugação de $11.000 \mathrm{~g}$, como recomenda BIRGEL ${ }^{1}$ (1969).

O volume corpuscular médio (VCM), a hemoglobina corpuscular média (HCM) e a concentração hemoglobínica corpuscular média (CHCM), foram calculadas conforme preceitua WINTROBE ${ }^{21}$ (1962).

$O$ sangue sofrendo tratamento idêntico ao utilizado para a contagem de hemácias, era coletado em pipetas hematimétricas de Thomas, para leucócitos onde, seguindo-se a técnica preconizada fazia-se a contagem dos leucócitos.

As contagens diferenciais de leucócitos foram feitas em esfregaços sanguíneos cora- 


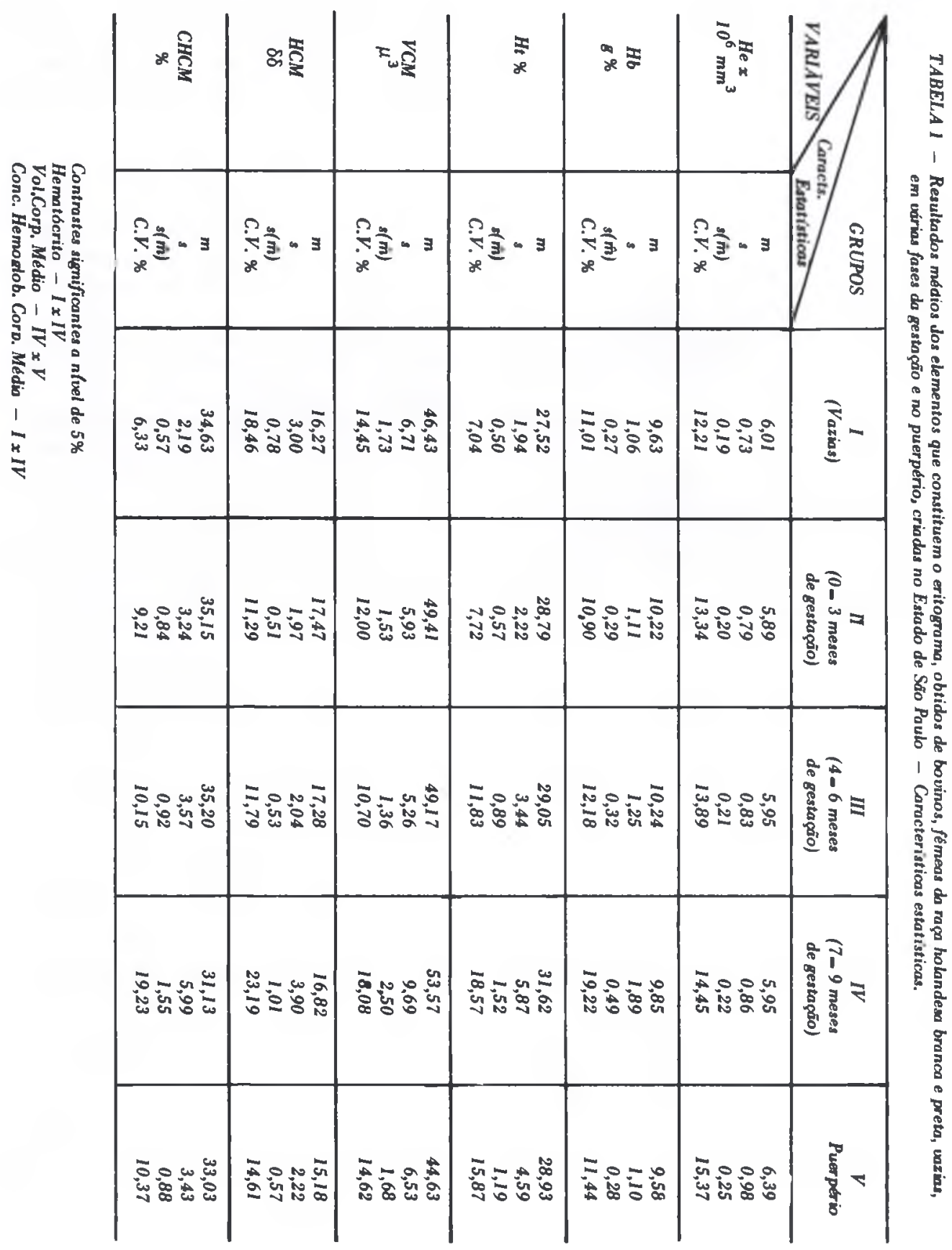




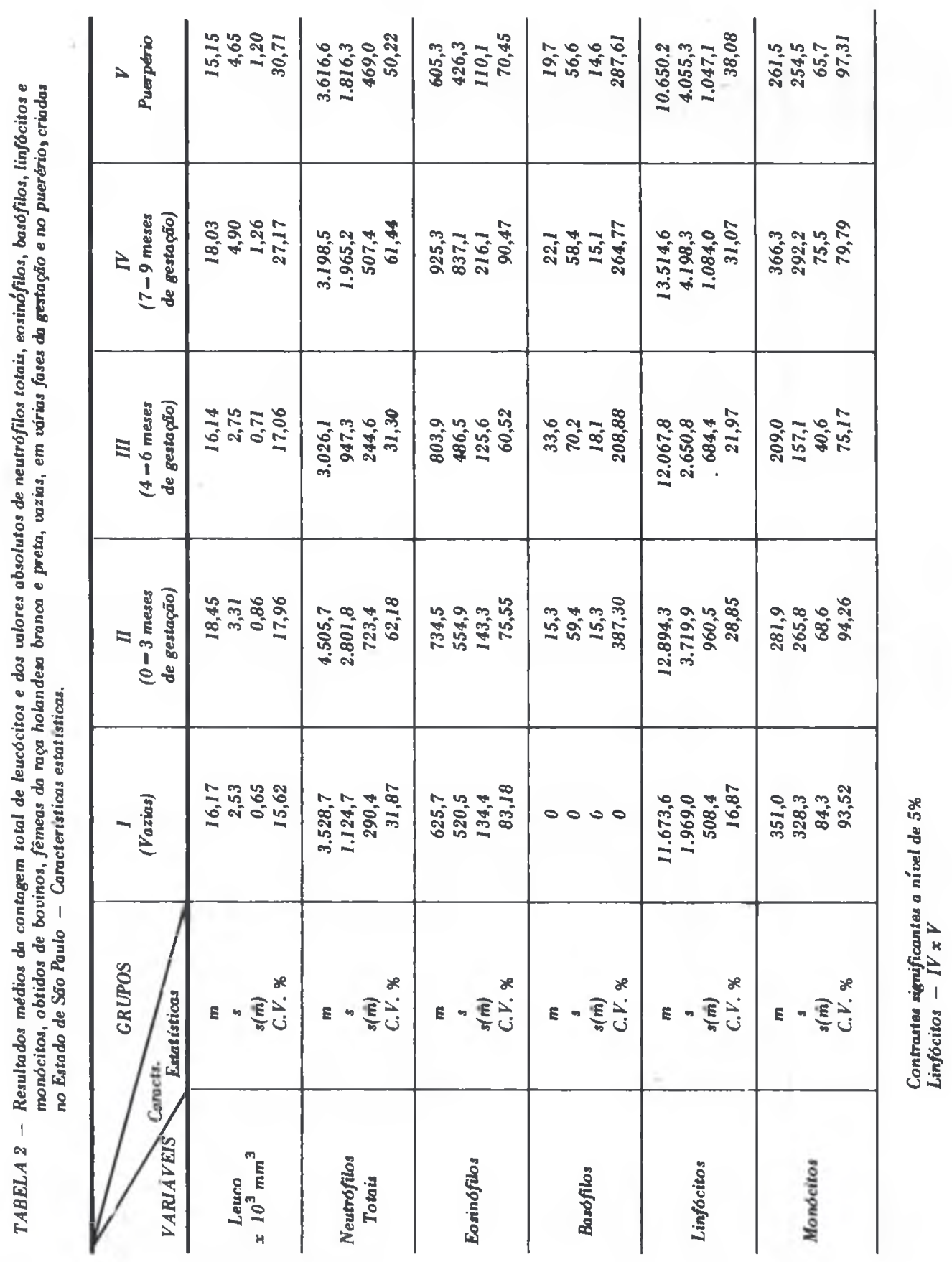

Rev. Fac. Med. vet. Zootec. Univ. S. Paulo 14(1):11-21, 1977. 


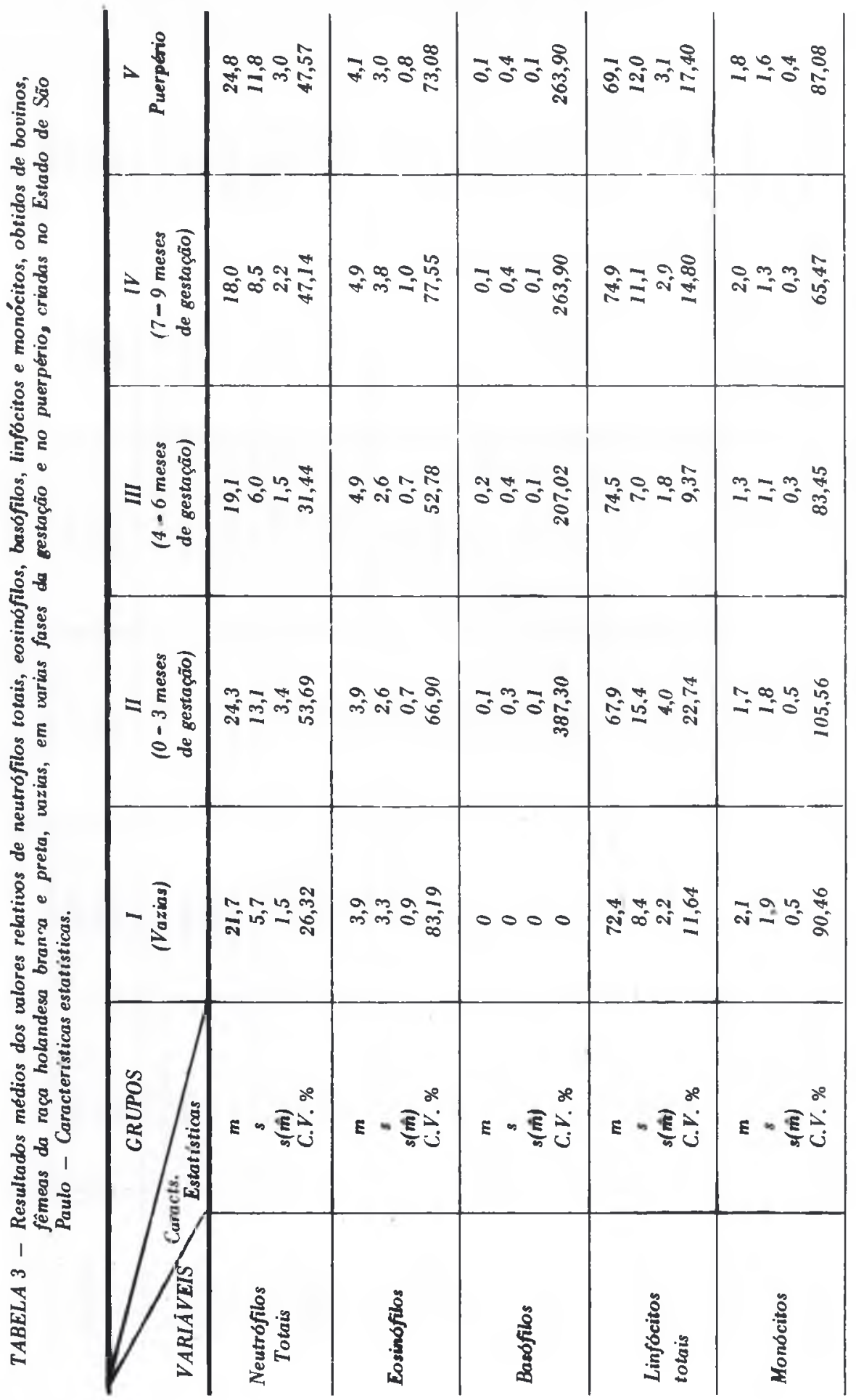


dos, utilizando-se a técnica preconizada por ROSENFELD $^{1415}$ (1947). A contagem foi feita sobre 100 células. Calculamos, além dos valores percentuais, os números totais para os diferentes tipos de leucócitos, em função da contagem global.

As verificaçōes da influência da gesta ção foram inicialmente testadas pela análise de variância (teste $\mathrm{F}$ ), seguindo-se o modelo de SNEDECOR ${ }^{18}$ (1956) e os contrastes entre as médias foram verificados pelo teste $T$ de Student, conforme recomenda GOMES ${ }^{6}$ (1963).

\section{RESULTADOS}

Os valores obtidos para a contagem de hemácias, os teores de hemoglobina, os valo. res de hematócrito, do VCM, do HCM, do CHCM, bem como para a contagem de leucócitos totais e para a contagem diferencial de leucócitos, nas várias fases da gestação e durante o puerpério estão consubstanciados nas tabelas 1, 2 e 3 . Além dos resultados médios obtidos para as variáveis estudadas nos diferentes grupos experimentais figuram nestas tabelas as características estatísticas representadas por : $\mathrm{s}$ (desvio padrão), $\mathrm{s}(\hat{\mathrm{m}})$ (desvio padrão da média), c.v.\% (coeficiente de variação de Pearson).

\section{DISCUSSÃO}

Ao se fazer uma apreciação dos resultados referidos pelos vários autores consultados, verifica-se que as conclusões apresentadas estão fundamentadas em amostragens insuficientes, deficientes ou mal definidas. Em vista disso, no planejamento da presente pesquisa, uma série de cuidados foram tomados, para apresentarem-se valores populacionais representativos do quadro hemático de vacas em gestação.

A homogeneidade da amostragem foi conseguida através da padronização da coleta do material, feita à mesma hora, após o exame clínico dos animais, sendo os mesmos só incluidos definitivamente no experimento depois do exame parasitológico das fezes, que os revelasse isentos de endoparasitoses, além de escolhermos animais numa faixa etária o mais estreita possível, além da vigilância para que houvesse uma constância quanto à alimentação e manejo durante todo o experimento.

No pertinente aos valores do número de hemácias, os nossos resultados não mostraram significado estatístico. Entretanto não concordam com os de MORRIS ${ }^{10}$ (1944) e $\operatorname{ELECKO}^{5}$ (1967), que verificaram, no puerpério uma diminuição do número de hemácias, bem como aos de CONNER et alii $^{4}$ (1967), que observaram uma diferença significante, devido à diminuição do número de hemácias com o decorrer da gestação.

No tocante aos teores de hemoglobina, em gramas por cento, nossos resultados não foram estatisticamente significantes, sendo assim, semelhantes aos achados de PATTERSON et alii $^{12}$ (1960) e ELECKO ${ }^{5}$ (1967) e discordantes dos achados de MORRIS $^{10}$ (1944). Para os valores do hematócrito constatamos um aumento significante durante todo o periodo de gestação para no puerpério diminuir de forma acentuada. Nossos resultados concordam com os VAN SOEST \& BLOSSER ${ }^{20}$ (1954) e LANE \& CAMPELL $^{9}$ (1969) que salientaram que o hematócrito aumenta a partir do $6^{\circ}$ mês de gestação e que no puerpério seus valores são menores. Nossos achados discordam dos de REYNOLDS $^{13}$ (1953) que em vacas primíparas, verificou não haver variações do volume globular durante toda a gestaçăo.

Os resultados obtidos para os índices hematimétricos absolutos, volume corpuscular médio (VCM) e concentração hemoglobínica corpuscular média, revelam que houve influência sistemática e significante dos fato. res de variação por nós estudados. Tendo em vista, não encontrarmos na literatura especializada, alguns resultados que pudessem ser comparados com os nossos, dificultando assim um estudo crítico, nos limitaremos apenas a descrever o comportamento destes índices, nas condições de experimento por nós utilizadas.

O VCM aumenta com o progredir da gestação, sendo seu valor no final desta fase significantemente maior do que o observado nas fêmeas vazias. Este aumento explicaria fato similar já descrito para o hematócrito. No puerpério houve uma diminuição evidente do VCM, tendendo o resultado a se igualar 
ao observado nas novilhas não prenhes. Comportamento diverso apresenta o CHCM pois há diminuição significante de seus valores no final da gestação.

Para o HCM, apesar de nảo apresentar significado estatístico observamos que os animais no terço inicial de gestaçåo apresentaram valores maiores do que os observados no puerpério.

Apesar de não apresentarem um estudo completo e sistemático $a$ influência da gestação sobre o leucograma de fêmeas da espécie bovina foi ressaltada por Moberg (1955) citado por SCHALM ${ }^{17}$ (1964), STRAUB et alii ${ }^{19}$ (1959), OTSUKA \& NAKAJIMA $^{11}$ (1961), KHAJURIA \& RAZDAN $^{8}$ (1966) ELECKO ${ }^{5}$ (1967), CONNER et alii ${ }^{4}$ (1967) e BOSTEDT \& BERCHTOLD $^{3}$ (1968).

Da mesma forma que ressaltaram CONNER et aliii (1967) o número global de leucócitos, no presente experimento, não sofreram a influência da gestação e do puerpério, pois as diferenças encontradas nos diferentes grupos nảo foram estatisticamente significantes. Os nossos resultados foram semelhantes aos obtidos por STRAUB et alii ${ }^{19}$ (1959) e ELECKO $^{5}$ (1967), por serem maiores, discordam dos resultados apresentados por KHAJURIA \& RAZDAN ${ }^{8}$ (1966) e CONNER et alii ${ }^{4}$ (1967). Convém destacar ainda que os menores valores obtidos para a contagem global de leucócitos foram obtidos no puerpério, dados estes concordantes com os de STRAUB et alii ${ }^{19}$ (1959).

Para os neutrófilos, no que se refere à contagem relativa, a análise de variância não mostrou diferenças estatisticamente significantes entre os 5 grupos estudados. Nossos valores, quando comparados com os dados constantes da literatura, se assemelham aos encontrados por KHAJURIA \& RAZDAN ${ }^{8}$ (1966) e CONNER et alii ${ }^{4}$ (1967). Por outro lado discordam das de Moberg (1955) citado por SCHALM ${ }^{17}$ (1964) que verificou aumento dos neutrófilos até o $6^{\circ}$ mês de gestação, para depois haver uma diminuição para novamente aumentar próximo ao parto, fato também verificado por ELECKO $^{5}$ (1967).

Os eosinófilos apresentaram ligeiras oscilações no decorrer de nossas observações, a análise estatística não revelou significância para os valores absolutos e relativos, nos 5 grupos estudados, fato que também foi comprovado por Moberg (1955) citado por SCHALM $^{17}$ (1964) e CONNER et $\mathrm{alii}^{4}$ (1967). No entanto, STRAUB ${ }^{9}$ (1959), OTSUKA \& NAKAJIMA ${ }^{1}$ (1961) BOSTEDT \& BERCHTOLD ${ }^{3}$ (1968) observaram uma eosinopenia no momento do parto, para voltar a níveis normais no puerpério, fato este năo constatado por nơs.

No que se refere aos basófilos, verificamos que os autores não se preocuparam em estudar este elemento da série branca, provavelmente devido a sua baixa freqüência, de fato obtivemos os seguintes valores médios: para a contagem relativa $0,1 \%$ e para a abso. luta $18,2 / \mathrm{mm}^{3}$.

Os resultados obtidos para os linfócitos também não mostraram diferenças estatisticamente significantes. Concordando com as nossas observaçôes, CONNER et alii ${ }^{4}$ (1967) não observaram modificações substanciais, durante a gestação. KHAJURIA \& RAZDAN $^{8}$ (1966) encontraram em novilhas prenhes, valores ligeiramente inferiores aos nossos.

A média dos valores relativos e absolutos de monócitos năo mostrou diferenças significantes entre os grupos estudados, fato esse também comprovado por STRAUB et alii $^{19}$ (1959), CONNER et alii (1967), e ELECKO $^{5}$ (1967), discordando entretando dos valores obtidos por KHAJURIA \& RAZDAN $^{8}$ (1966).

\section{CONCLUSÕES}

0 estudo do quadro hemático de fêmeas jovens, da espécime bovina, da raça holandesa branca e preta, em várias fases de gestação e durante o puerpério, permitem as seguintes conclusões:

1 - 0 delineamento experimental proposto permite verificar que a gestação influe sobre o volume globular e volume corpuscular médio (VCM), havendo aumento estatisticamente significante do volume médio das hemácias, responsável direto pelo maior valor hematócrito no final da gestação. De maneira con- 


\begin{tabular}{|c|c|c|c|c|c|c|c|c|c|c|c|c|}
\hline 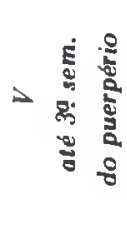 & $\begin{array}{l}\approx \\
0 \\
+1 \\
0 \\
0 \\
0 \\
0\end{array}$ & $\begin{array}{l}2 \\
= \\
+1 \\
1 \\
5 \\
2\end{array}$ & $\begin{array}{l}\text { के } \\
+ \\
+1 \\
2 \\
\infty \\
\text { के }\end{array}$ & $\begin{array}{l}n \\
0 \\
0 \\
+1 \\
\ddot{0} \\
\ddot{0} \\
+\end{array}$ & 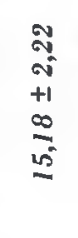 & 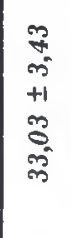 & $\begin{array}{l}0 \\
0 \\
+1 \\
10 \\
10 \\
5\end{array}$ & 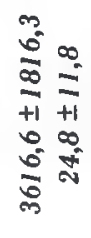 & 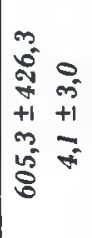 & 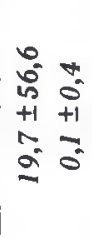 & 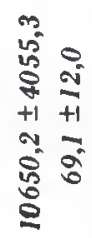 & 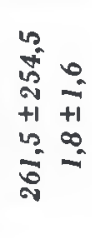 \\
\hline 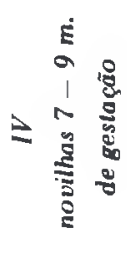 & $\begin{array}{l}0 \\
0 \\
0 \\
+1 \\
2 \\
\text { is }\end{array}$ & $\begin{array}{l}\infty \\
\infty \\
+1 \\
+1 \\
\infty \\
\infty \\
0\end{array}$ & 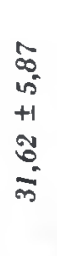 & $\begin{array}{l}8 \\
0 \\
+1 \\
i \\
i s \\
\text { in } \\
\text { in }\end{array}$ & $\begin{array}{l}8 \\
\infty \\
+1 \\
0 \\
0 \\
0 \\
0\end{array}$ & $\begin{array}{l}2 \\
2 \\
+1 \\
+1 \\
2 \\
=\end{array}$ & $\begin{array}{l}8 \\
\alpha \\
+1 \\
+1 \\
0 \\
0 \\
0\end{array}$ & 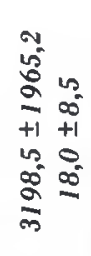 & 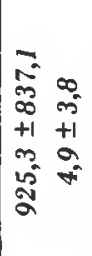 & 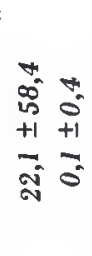 & 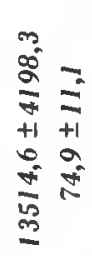 & 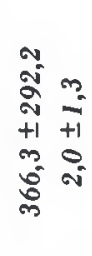 \\
\hline 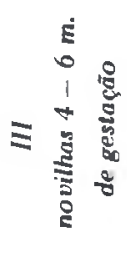 & $\begin{array}{l}\approx \\
0 \\
0 \\
+1 \\
20 \\
2 \\
\text { is }\end{array}$ & 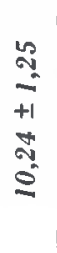 & 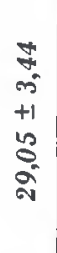 & \begin{tabular}{l} 
o \\
is \\
+1 \\
$\vdots$ \\
$\vdots$ \\
\multirow{2}{*}{}
\end{tabular} & 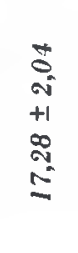 & 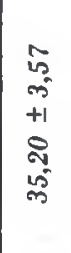 & $\begin{array}{l}n \\
a \\
0 \\
+1 \\
\Delta \\
0 \\
0\end{array}$ & 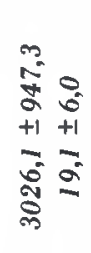 & 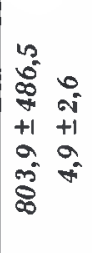 & 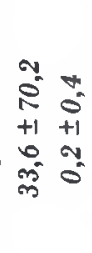 & 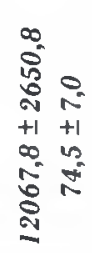 & 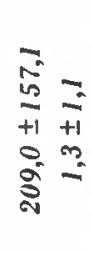 \\
\hline 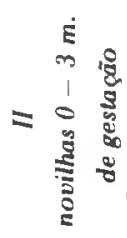 & $\begin{array}{l}2 \\
0 \\
0 \\
+1 \\
0 \\
0 \\
i\end{array}$ & 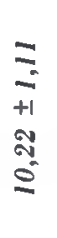 & 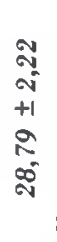 & $\begin{array}{l}2 \\
\text { is } \\
+1 \\
j \\
\vdots\end{array}$ & $\begin{array}{l}\hat{a} \\
+1 \\
\vdots \\
\vdots \\
=\end{array}$ & 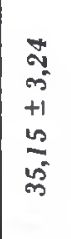 & $\begin{array}{l}m \\
m \\
+1 \\
3 \\
\vdots \\
\infty \\
0\end{array}$ & 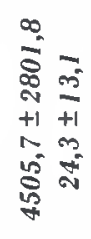 & 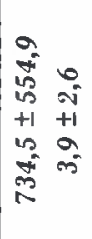 & $\begin{array}{ll}* & m \\
\text { in } & 0 \\
+1 & +1 \\
0 & 0 \\
\text { is } & 0\end{array}$ & 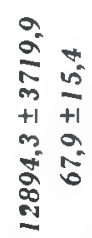 & 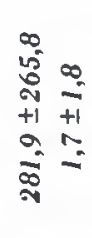 \\
\hline \multirow[t]{2}{*}{ 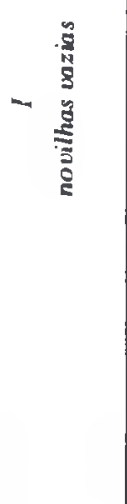 } & $\begin{array}{c}2 \\
0 \\
+1 \\
0 \\
0 \\
0\end{array}$ & 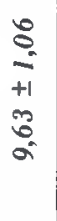 & 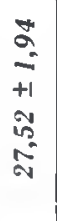 & $\begin{array}{l}\overline{1} \\
6 \\
+1 \\
3 \\
\vdots \\
0\end{array}$ & \begin{tabular}{l}
8 \\
के \\
+1 \\
\multirow{2}{1}{} \\
0
\end{tabular} & $\begin{array}{l}a \\
\text { oे } \\
+1 \\
\ddot{8} \\
\dot{0} \\
\text { oे }\end{array}$ & \begin{tabular}{l} 
ro \\
\multirow{2}{*}{} \\
+1 \\
+1 \\
$=$ \\
0
\end{tabular} & 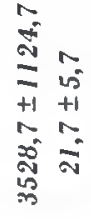 & 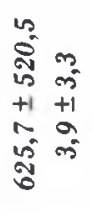 & 00 & 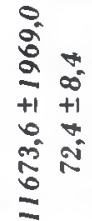 & 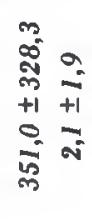 \\
\hline & 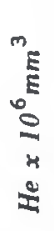 & 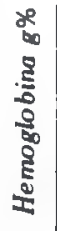 & 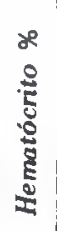 & 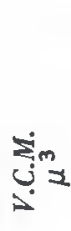 & خيخ & نم & 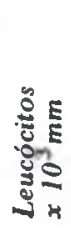 & 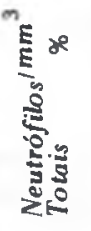 & 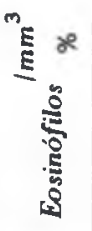 & 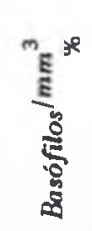 & 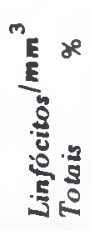 & 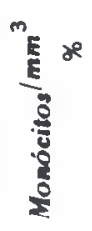 \\
\hline
\end{tabular}

Rev. Fac. Med. vet. Zootec. Univ. S. Paulo, 14(1):11-21, 1977. 
trária, por permanecerem inalteradas as taxas de hemoglobina observou-se uma diminuição estatisticamente significante da concentração de hemoglobina corpuscular média (CHCM) no final da gestação.

2 - O número de hemácias e os teores de hemoglobina não sofrem influências estatisticamente significantes da gestação e do puerpério.
3 - 0 quadro leucocitário não sofre influências estatisticamente significantes da gestação e do puerpério.

4 - Os valores dos elementos que constituem o hemograma para fêmeas bovinas da raça holandesa branca e preta não prenhes, em várias fases da gestação e no puerpério, expressos em valo. res médios e desvio padrão da média são os seguintes:

\section{RFMV-A/ I}

D'ANGELINO, J.L.; ARAUJO, L.M.; BIRGEL, E.H.; ARAUJO, W.P.; REICHMANN, C.E. Influence of pregnancy and puerperium on the blood picture of white and black Dutch breed bovines. Rev. Fac. Med. vet. Zootec. Univ. S.Paulo, 14(I): 11.21. 1977.

SUMMARY: The variances in the blood picture of bovines ingestation were studied in 75 black and white Dutch breed heifers. At the end of the gestation period there was observed a significtunt increase of the MCV responsible for the increase of the PCV. Because the hemoglobin values were unaltered a significant decrease of $M C H C$ was noticed at the end of gestation period. The leucocyles and differential couting showed no variations during gestation period and recent puerperium. The results obtained for the group of empty heifers, animals in their initial gestation phase, between 4 and 6 months of gestation, heifers at the end of their gestation and for cows during their puerperium period were respectivelly as follow:

Erythrocytes (million/cu mm) - 6,01; 5,89; 5,95; 5,95 e 6,39

Hemoglobin (gm/lo0cc) - 9,63; 10,22;10,24; 9,85 e 9,58

$P C V(\%)-27,52 ; 28,79 ; 29,05 ; 31,62$ e 28,93

$M C V(c u \mu)-46,43 ; 49,41 ; 49,17 ; 53,57$ e 44,63

$\mathrm{MCH}(\mathrm{rgm})-16,27 ; 17,47 ; 17,28 ; 16,82$ e 15,18

MCHC (\%) - 34,63; 35,15; 35,20; 31,13 e 33,03

Leucocytes (thousand/cu mm) - 16,17;18,45;16,14;18,03 e 15,15:

neutrophil (\%) $-21,7 ; 24,3: 19,1 ; 18,0$ e 24,8

(cu mm) - 3528,7; 4505,7; 3026,1; 3198,5 e 3616,6

eosinophil (\%) $-3,9 ; 3,9 ; 4,9 ; 4,9 e 4,1$; th

(cu mm) - 62.5.7; 734,5; 80.3,9; 925,3 e 605,3

basophil $(\%)--0 ; 0,1 ; 0,2 ; 0,1$ e 0,1

(cu mm) - $0 ; 15,3 ; 33,6 ; 22,1$ e 19,7

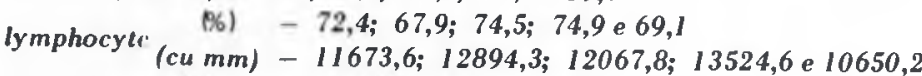

monocyte $(\%)-2,1 ; 1,3 ; 1,3 ; 2,0$ e 1,8

(cu $\mathrm{mm})-351,0 ; 281,9 ; 209,0 ; 366,3$ e 261,5

UNITERMS: Puerperium *; Pregnancy, bovine *; Hematology *; Blood picture.

\section{REFERÊNCIAS BIBLIOGRĀFICAS}

1 - BIRGEL, E.H. Métodos de diagnóstico em hematologia clínica. In: BIRGEL, E.H. et alii, ed. Meios e métodos de diagnớstico em medicina veterinária. São Paulo, 1969. p.9-33. (Multilitado)

2 - BOROVICZENY, G.H.G. Comentos on the decision to standardize haemoglo- binometry in reytrocytometric methods and their standardization. In: CONGRESS OF THE EUROPEAN SOCIETY OF HA EMA TOL OGY, 9. Lisbon, 1963. Proceendings. Basel, S.Karger, 1964. p.112*18.

3 - BOSTEDT, H. \& BERCHTOLD, M. Blo. 
od glucose and eosinophile count cow during and after parturition. Berl. Munch. tierarztl. Wschr., 81: 243-45, 1968.

4 - CONNER, G.H.; LABELL, J.A.; EYSTER, J.; WONNACOTT, V. Effects of pregnancy and age on hemograms of Holstein friesian in herd with no evidence of leukemia. Amer. J. vet. Res., 28: 1303-12, 1967.

5 - ELECKO, J. Changes in the blood picture of cow before, during and after parturition. Folia vet., 11(2): $139-49,1967$.

6 - GOMES, F.P. Curso de estatística experimental. 2.ed. Piracicaba, 1963.

7 - KAMPEN, E.J.van \& ZIJLSTRA, W.G. Standardization of haemoglo. binometry. In: CONGRESS OF THE EUROPEAN SOCIETY OF HAEMATOLOGY, 9, Lisbo n, 1963 . Proceendings. Basel, S.Karger, 1964. p.68-72.

8 - KHAJURIA, R.R. \& RAZDAN, M.N. Hematological studies in dairy cattle. II. Leucocytic picture under different physiological states. Indian vet. J., 43: 886-92, 1966.

9 - LANE, A.G. \& CAMPELL, J.R. Relationship of hematocrit values to selected physiological conditions in dairy cattle. J. Animal Sci., 4: 508-11, 1969.

10 - MORRIS, P.G.D. The blood picture of a cow during a normal pregnancy and parturition. Brit. vet. Sci., 100: 225,1944

11 - OTSUKA, J. \& NAKAJIMA, T. Changes in circulating eosinophiles during growth and pregnancy in cattle. Bull. Nat. Inst. agr. Sci., Tokio, 6(20): $263-67,1961$.

12 - PATERSON, T.B.; SHRODE, R.R.; KUNKEL, H.D.; LEIGHTON, R.E.; RUPEL, I.W. Variations in certain blood components of holstein and Jersey cows and their relationship and to daily range in nectal temperature and to milk and butterfat production. J. Dairy Sci, 43: 1263, 1960.

13 - REYNOLDS, M. Measurements of bovine plasma and blood volume during pregnancy and lavtation Amer. J. Physiol., 175: 188, 1953.

14 - ROSENFELD, G. Método rápido de coloração de esfregaços de sangue. Noções práticas sobre corantes pancrômicos $\mathrm{e}$ estudo de diversos fatores, Mem. Inst. Butantan, São Paulo, 20: 315 $-29,1947$.

15 - ROSENFELD, G. Corante pancrómico para hematologia e citologia clínica. Nova combinação dos componentes do May-Grunwald e do Giemsa num só corante de emprego rápido. Mem. Inst. Butantan, São Paulo, 20: 329-34, 1947.

16 - ROSENFELD, G. Etilenodicmina tetracitica dissodica (EDTA) como anticoagulante para técnica hematológica. Rev. clin. São Paulo, 31: 65-71, 1955.

17 - SCHALM, O.W. Veterinary hematology 2.ed. Philadelphia, Lea \& Febiger, 1964.

18 - SNEDECOR, G.W. Statistical methods. 5.ed. Iowa, Iowa State College Press, 1956.

19 - STRAUB, O.C.; SCHALM, O.W.; HUGHES, J.P.; THEILEN, G.H. Bovine hematology, II. Effect of parturition and retartion of fetal membranes on blood morphology. J. Amer. vet. med. Ass., 135: 618, 1959.

20 - VAN SOEST, P.J. \& BLOSSER, T.H. A detailed study of levels of certain blood constituints in normally calving dairy $\infty$ ws and in dairy cows with parturient paresis. J. Dairy Sci., 37: $185,1954$.

21 - WINTROBE, M.M. Clinical hematolo. gy. 5.ed. Philadelphia, Lea \& Febiger, 1962.

Recebido para publicação em 5-5-77 Aprovado para publicação em 3-8-77

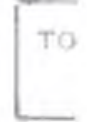

